
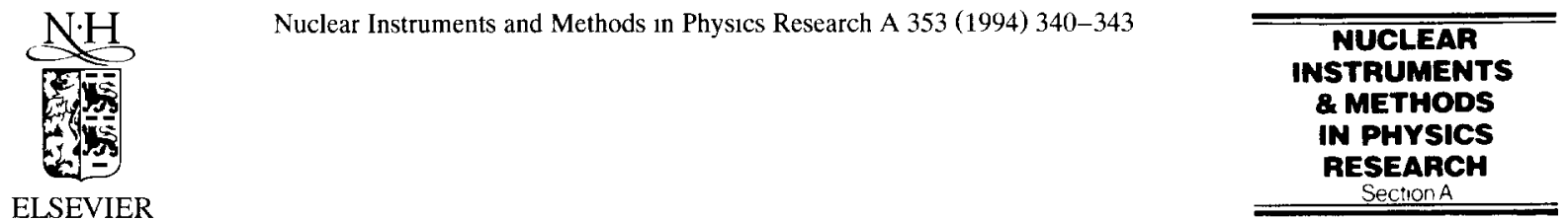

ELSEVIER

\title{
Background-adaptive dual-energy-window correction for Compton scattering in SPECT
}

\author{
Jian-qiao Luo *, Kenneth F. Koral \\ Dilısıon of Nuclear Medicine, University of Michigan Medical Center, Ann Arbor, MI 48109-0552, USA
}

\begin{abstract}
Detection of gamma rays which Compton scatter within a patient but are still within the photopeak window of the Anger camera leads to inaccuracies in quantification of radioactivity from nuclear-medicine images. With the dual-energy-window correction method and a single (universal) scatter multiplier, the activity error is relatively large when there is tissue background with a large range of values including zero. We examine here a procedure that adapts the scatter multiplier to the level of background. In a Monte Carlo investigation, we introduce the iterative technique, examine when it converges, and look at the resultant improvement in quantification. Three geometries are checked: a large-sphere ${ }^{99 \mathrm{~m}} \mathrm{Tc}$ target within a cylinder containing 1) a uniform or 2) non-uniform background and 3) a ${ }^{123} \mathrm{I}$ brain phantom. Reconstruction of the data is carried out with the iterative maximum-likelihood, expectation-maximization algorithm with attenuation correction. Results show that the multiplier converges to a stable value after only a few iterations for all cases. Typical errors in target activity are: for the off axis sphere in non-uniform background $23.3 \%$ (no correction), $-13.9 \%$ (universal-multiplier correction), and $-0.7 \%$ (converged-multiplier correction); for the putamen in uniform white-matter background $20.4 \%$ (no correction), $-10.6 \%$ (universal-multiplier correction), and $3.0 \%$ (converged-multiplier correction). The iterative background-adaptive method leads to considerable improvement in all cases tested.
\end{abstract}

\section{Introduction}

In general, detection of gamma rays which Compton scatter within a patient but are still within the photopeak window of the Anger camera leads to inaccuracies in quantification of radioactivity from nuclear-medicine single-photon emission computed tomography (SPECT). The dual-energy-window correction method [1,2], in which counts in a lower-energy window are multiplied by a "scatter multiplier" and subtracted from those in the photopeak window, is simple, and Monte Carlo investigations indicate that in ${ }^{99 \mathrm{~m}} \mathrm{Tc}$ SPECT of focal, radioactive regions, correct activities can be obtained. A single (universal) scatter multiplier is fairly accurate in the face of variations in patient size and in location of the focal activity. However, the activity error is much larger for the cases where variation in tissue background has a large range of values including zero $[3,4]$.

We examine here a procedure that adapts the scatter multiplier to the level of background. In a Monte Carlo

\footnotetext{
* Corresponding author.
}

investigation, we 1) introduce the iterative technique, 2) examine when it converges, and 3) look at the resultant improvement in quantification. Complications from high levels of statistical noise are expected to be small but are not considered.

\section{Method}

In order to account for the problems of background, we propose an iterative correction technique as follows: 1) obtain, from a phantom or Monte Carlo experiment, a curve of correct scatter multiplier versus uniform background level for a geometry which is similar to those of interest; 2) use the zero-background scatter multiplier to form the zeroth-iteration corrected image set for the test object; 3) from the image set, determine the ratio of average background-activity concentration over target-activity concentration. At this value on the guide curve, read off a new (first-iteration) scatter multiplier; 4) use the first-iteration scatter multiplier to form the first-iteration corrected image set for the test object. Step 4 is the same as step 2 except that the scatter multiplier and iteration number have changed. Therefore, we have defined an iteration loop. If the scatter multiplier is converging toward 


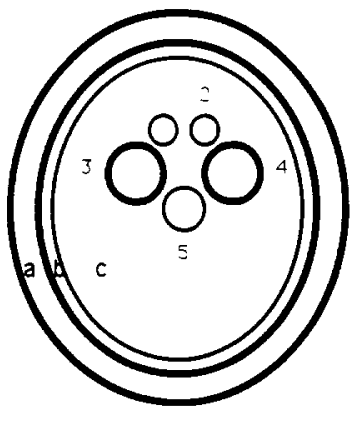

Fig. 1. A cross section through the three dimensional brain phantom. Here, a marks the highly-attenuating skull, $b$ the cortical region, $\mathrm{c}$ the white-matter background region and the numbers denote the sub-cortical nuclei which are modeled by spheres. In particular, 1 and 2 are the caudate nuclei, 3 and 4 the putamen and 5 the thalamus.

a single value, iteration is stopped when the change is less than a preset percentage.

Three geometries are checked in this preliminary investigation. One is a large-sphere ${ }^{99 \mathrm{~m}} \mathrm{Tc}(141 \mathrm{keV})$ target within a circular cylinder containing a uniform background. The diameter and length of the cylinder are 20.5 and $23.8 \mathrm{~cm}$. The diameter of the sphere is $5.7 \mathrm{~cm}$ and it is located either on the axis of the cylinder or $5.7 \mathrm{~cm}$ off axis. In either case, it is at the midpoint of the length. The SPECT transverse reconstructions cut the cylinder parallel to the ends. The second is similar but with a non-uniform background. That is, the target sphere is $5.7 \mathrm{~cm}$ off axis and three other spheres are that same distance off axis and located so the four are arranged symmetrically. The three are identical to each other as far as activity. There is now no activity in the cylinder. The third is an ${ }^{12.3} \mathrm{I}(159 \mathrm{keV})$ brain phantom (a sketch to scale is given in Fig. 1) with several spheres simulating the centrally-located sub-cortical nuclei and with white matter throughout the elliptical cylinder and a cortical annulus just inside the skull. Attenuation is now non-uniform because the skull is more highly attenuating than the other tissues.

In this study, the photopeak window is $126-154 \mathrm{keV}$ and the scatter window is $98-126 \mathrm{keV}$ for ${ }^{99 \mathrm{~m}} \mathrm{Tc}$ and 143.1-174.9 keV and 111.3-143.1 keV for ${ }^{123} \mathrm{I}$. Also, the Compton-scatter correction is carried out on image sets of three phantoms reconstructed from the photopeak-window projections and the scatter-window projections. Reconstruction of the corrected data is accomplished with the iterative maximum-likelihood, expectation-maximization algorithm. Maps for attenuation correction are the same as those used to generate the simulated data. Sixteen iterations are sufficient for stable average counts within a volume of interest $(\mathrm{VoI})$. For tests with the uniform-background phantom, the ratio of cylinder-activity concentration over sphere-activity concentration equals 0.2 . For the non-uniform-background phantom, the ratio of activity concentration in each of the three spheres over that in the target sphere is 5.5 which makes the average concentration in the volume outside the target sphere over the concentration in the target sphere equal to 0.2 . For the brain phantom, the relative activity concentrations for the caudate nuclei, putamen, thalamus, cortex and white-matter background are $25,25,12,4$, and 1 respectively. These values are typical for an IBVM brain scan at 4 hours after injection [5]. They make the ratios for background concentration over target concentration equal $0.04,0.04,0.08$, and 0.25 for the four targets in the order given above. The same multiplier-versus-background guide curve is employed throughout this study. It is the Monte Carlo curve for a ${ }^{99 m} \mathrm{Tc}$ on-axis sphere in a uniform background cylinder. Sizes are the same as those used here. A plot of the curve is given in Ref. [3] and, along with the exact values, in Ref. [4]. For the computations of activity, the target $\mathrm{VoI}$ is congruent with the true geometric volume of the target object. The error is obtained by taking the difference between the reconstructed counts in the Vol for the image set from the corrected data and the reconstructed counts in the same VoI for the image set from the unscattered photons in the photopeak window. It is converted to a percent by dividing by the counts from the unscattered photons.

\section{Results}

Table 1 shows the scatter multiplier as a function of iteration number for the sphere-within-a-circular-cylinder phantoms. It shows that the multiplier converges to a stable value after only a few iterations for all three cases. The approach is monatomic for uniform background and has a slight undershoot with return to final value for non-uniform. For comparison, the multiplier which produces zero error is also listed.

Table 2 shows the error in target activity for the same cases. There is a large improvement in activity quantification from using the converged scatter multiplier compared

Table 1

Convergence of scatter multiplier for sphere located on and also off axis with uniform and non-uniform background

\begin{tabular}{|c|c|c|c|}
\hline \multirow{2}{*}{$\begin{array}{l}\text { Background } \\
\text { Sphere location }\end{array}$} & \multicolumn{2}{|l|}{ Uniform } & \multirow{2}{*}{$\begin{array}{l}\text { Non-uniform } \\
\text { off-axis }\end{array}$} \\
\hline & on-axis & off-axis & \\
\hline \multicolumn{4}{|l|}{ Iteration } \\
\hline 0 & 1.251 & 1.251 & 1.251 \\
\hline 1 & 0.862 & 0.868 & 0.795 \\
\hline 2 & 0.824 & 0.829 & 0.808 \\
\hline 3 & 0.822 & 0.826 & 0.808 \\
\hline 4 & 0.821 & 0.825 & 0.808 \\
\hline $\begin{array}{l}\text { Multiplier required for } \\
\text { error-free activity } \\
\text { estimate }\end{array}$ & 0.815 & 0.778 & 0.784 \\
\hline
\end{tabular}


Table 2

Activity error for sphere with three different multipliers

\begin{tabular}{lccc}
\hline Background & \multicolumn{2}{l}{ Uniform } & \multirow{2}{*}{$\begin{array}{l}\text { Non-uniform } \\
\text { off-axıs }\end{array}$} \\
\cline { 2 - 3 } Sphere location & on-axis & off-axis & \\
\hline Multiplier & & & \\
Zero & $25.658 \%$ & $22648 \%$ & $23.282 \%$ \\
Universal & -13.727 & -13.753 & -13.882 \\
Converged & -0.189 & -1.367 & -0.713 \\
\hline
\end{tabular}

Table 3

Convergence of scatter multiplier for sub-cortical nucles and cortex with white-matter background

\begin{tabular}{lllll}
\hline Iteration & Caudate & Putamen & Thalamus & Cortex \\
\hline 0 & 1251 & 1.251 & 1.251 & 1.251 \\
1 & 0.650 & 0.725 & 0.606 & 0.516 \\
2 & 0.634 & 0.702 & 0.601 & 0.513 \\
3 & 0.634 & 0.701 & 0.601 & 0.513 \\
4 & 0.634 & 0.701 & 0.601 & 0.513 \\
$\begin{array}{l}\text { Multiplier required } \\
\text { for error-free }\end{array}$ & 0790 & 0.824 & 0.756 & 0.601 \\
$\quad$ & & & & \\
\hline
\end{tabular}

to using the universal (zeroth iteration) multiplier. All activities are slightly too small, but there is a large improvement in each case. The best result $(-0.189 \%)$ occurs when the test case is completely consistent with the guide curve. The error with a zero multiplier (no correction for Compton scatter at all) is also shown for comparison.

Table 3 shows the scatter multiplier as a function of iteration number for the brain phantom. Convergence is again very rapid and the approach is monatomic in all cases. The multiplier which produces zero error is also given.

Table 4 shows the error in target activity for the sub-cortical nuclei and the cortex. All activities are too large with no correction, too small with the universal multiplier, and end up slightly too large. The cortex shows the largest change with iteration (from $-24.1 \%$ to $3.28 \%$ ). The thalamus has the biggest final error $(6.09 \%)$; however, it had the largest universal-multiplier error of the three sub-cortical nuclei.

Table 4

Activity error for targets in brain phantom with three different multıpliers

\begin{tabular}{lllll}
\hline $\begin{array}{l}\text { Target } \\
\text { Multipliers }\end{array}$ & caudate & putamen & thalamus & cortex \\
\hline Zero & $24.41 \%$ & $20.44 \%$ & $29.73 \%$ & $22.29 \%$ \\
Universal & -14.23 & -10.61 & -19.45 & -24.10 \\
Converged & 4.84 & 3.05 & 6.09 & 3.28 \\
\hline
\end{tabular}

\section{Discussion}

In the more complicated phantoms, there are interpretational decisions which have to be made to operate the algorithm. Consider the four-sphere-plus-cylinder phantom. Three of the spheres provide the background activity but by which volume should they be divided to yield concentration? By their own or that of the cylinder? The answer is provided by comparing to the geometry for the guide multiplier-versus-background curve: a target sphere with background activity in a cylinder. One should, for background concentration, take all the activity within the cylinder excluding the target activity and divide by the total volume of the cylinder excluding the target volume.

Now consider the brain phantom. In the case of the brain phantom when one of the sub-cortical nuclei or the cortex was considered as the target, the background was distributed over the white matter and activity in the other nuclei ignored. This is clearly a large simplifying approximation which introduces considerable error in $k$, but yields an acceptable small error in the activity estimates as seen in Tables 3 and 4.

In looking to quantify targets in the brain phantom, a calibration curve with ${ }^{123}$ I would probably have yielded better results. Nevertheless, testing convergence of all hot objects with a single guide curve seemed like a good exercise. Moreover, it appears that the dependence on background is of about the same general character in many cases. We infer this conclusion because results from the brain phantom were relatively good and because the edge cortical activity and the more internal nuclei activity both had similar final error. Other simple combinations of object and guide curve are, however, deserving of further investigation.

\section{Conclusion}

The iterative technique designed to adapt the Compton-scatter-correction multiplier for focal activity to the level of surrounding background works. Using a single

${ }^{99 \mathrm{~m}} \mathrm{Tc}$ guide curve, the method quickly converged for the seven target objects tested. Moreover, for the ${ }^{99 \mathrm{~m}} \mathrm{Tc}$ targets, the errors in the focal activity were reduced from more than $13 \%$ with the universal multiplier to less than $2 \%$. For the ${ }^{123} \mathrm{I}$ targets, the errors decreased from as much as $24.1 \%$ with the universal multiplier to between $3.05 \%$ and $6 \%$.

\section{Acknowledgements}

The authors thank Carey E. Floyd and Ronald J. Jaszczak, Department of Radiology, Duke University, for providing one Monte Carlo simulation program and Michael Ljungberg, Department of Radiation Physics, Unı- 
versity of Lund, Sweden, for another. This work was supported by PHS Grant Number R01 CA38790 awarded by the National Cancer Institute, DHHS. Its contents are solely the responsibility of the authors and do not necessarily represent the official views of the National Cancer Institute.

\section{References}

[1] R. Jaszczak, K. Greer, C. Floyd, C. Harris and R. Coleman, J. Nucl. Med. 25 (1984) 983.
[2] K. Koral, F. Swailem, S. Buchbinder, N. Clinthorne, L. Rogers and B.M.W. Tsui, J. Nucl. Med. 31 (1990) 90.

[3] J. Luo, K. Koral, M. Ljungberg, C. Floyd and R. Jaszczak, A Monte-Carlo investigation of dual-energy-window scatter correction for volume-of-interest quantification in Tc-99m SPECT, submitted to Phys. Med. Biol.

[4] J. Luo, Dual window scatter correction in quantitative single photon emission computed tomography. A Doctoral Dissertation Biomedical Science, Medical Physics, Oakland University, Rochester, MI, USA (1993).

[5] D. Kuhl, R. Koeppe, J. Fessler, S. Minoshima, R. Ackermann, J. Carey, D. Gildersleeve, K. Frey and D. Wieland, J. Nucl. Med. 35 (1994) 405. 